\title{
RisaAligner software for aligning fluorescence data between Agilent 2100 Bioanalyzer chips: Application to soil microbial community analysis
}

\author{
Elisabeth Navarro ${ }^{1,2}$, Olivier Fabrègue ${ }^{1}$, Riccardo Scorretti ${ }^{1}$, Jérémy Reboulet ${ }^{1}$, Pascal \\ Simonet ${ }^{1}$, Lorna Dawson ${ }^{3}$, and Sandrine Demanèche ${ }^{1}$ \\ ${ }^{1}$ Université de Lyon, Laboratoire Ampère (CNRS UMR5005), Environmental Microbial Genomics, École \\ Centrale de Lyon, Ecully, France, ${ }^{2}$ IRD-UMR LSTM, Campus de Baillarguet, Montpellier, France, and \\ ${ }^{3}$ James Hutton Institute, Aberdeen, UK
}

\author{
BioTechniques 59:347-358 (December 2015) doi 10.2144/000114363 \\ Keywords: microbial ecology; RISA comparison; alignment tool
}

Supplementary material for this article is available at www.BioTechniques.com/article/114363.

Ribosomal Intergenic Spacer Analysis (RISA) is a high-resolution and highly reproducible fingerprinting technique for discriminating between microbial communities. The community profiles can be visualized using the Agilent 2100 Bioanalyzer. Comparison between fingerprints relies upon precise estimation of all amplified DNA fragment lengths; however, size standard computation can vary between gel runs. For complex samples such as soil microbial communities, discrimination by fragment size is not always sufficient. In such cases, the comparison of whole fluorescence data as a function of time (electrophoregrams) is more appropriate. When electrophoregrams [fluorescence $=f$ (time)] are used, and more than one chip is involved, electrophoregram comparisons are challenging due to experimental variations between chips and the lack of correction by the Agilent software in such situations. Here we present RisaAligner software for analyzing and comparing electrophoregrams from Agilent chips using a nonlinear ladder-alignment algorithm. We demonstrate the robustness and substantial improvement of data analysis by analyzing soil microbial profiles obtained with Agilent DNA 1000 and High Sensitivity chips.

Microbial ecology research has benefited from developments in metagenomics as numerous methods are now available to exploit microbial DNA directly extracted from the environment (1). Initially, metagenomics was limited to the cloning of recovered DNA into plasmids, fosmids, or BACs for constructing gene libraries in a host strain organism such as E. coli $(2,3)$. These approaches aimed to detect new genes either by screening the library clones using molecular methods such as PCR and hybridization (4-6) or by detecting their function using phenotypic screening for cloned genes that could be efficiently expressed in the heterologous host (7).
High throughput sequencing technologies for metagenomic DNA opened new avenues for evaluating the functional potential of bacterial communities by analyzing sequence reads on metagenomics-dedicated annotation platforms $(8,9)$. The same approach potentially can be applied to taxonomically characterize bacterial communities, but its sensitivity is limited by dilution of phylogenetic marker genes such as ribosomal genes, among other bacterial genes $(10,11)$. This limitation justifies the use of PCR to specifically amplify these genes.

The structure of ribosomal genes composed of taxonomically relevant variable regions flanked by highly conserved regions is suitable for defining universal PCR primers (12). 16S rRNA amplicon sequencing has become a reference for providing snapshots of environmental community taxonomical structures with improved sensitivity (13), but the approach is still time-consuming and expensive for routine application with many samples (14). Moreover, the large volume of data generated by high-throughput sequencing is not necessarily useful for observing bacterial community modifications.

As and alternative, PCR products can be analyzed by rapid, robust, and reproducible techniques for establishing size or sequence based band patterns that can easily be compared between samples.

\section{METHOD SUMMARY}

RisaAligner is a new software package for comparing microbial DNA profiles without DNA sizing that uses a nonlinear alignment algorithm to obtain perfect matches between ladder profiles from different Agilent chips relative to a reference ladder. A second manual alignment is included to correct intra-chip shifts, and two additional options for correcting negative values that have no biological meaning are possible. 
One of the most popular fingerprinting methods is based on the variable size of the intergenic region separating rRNA genes in bacteria, archaea, fungi, or diatoms, which can be easily amplified by PCR using primers designed for the appropriate conserved regions within the 2 genes (14-22). This technique, Ribosomal Intergenic Spacer Analysis (RISA), involves PCR fragment size determination, which can be carried out on a denaturing 5\% polyacrylamide gel (23-25), with the Agilent 2100 Bioanalyzer, or with an ABI Prism Genetic analyzer (18,21,22,26).

Although the Agilent 2100 Bioanalyzer cannot determine the size of fragments with the precision commonly obtained using DNA sequencers, it remains an affordable option when access to sequencing machines is not possible since it still offers improved speed, simplicity, and better resolution compared to traditional polyacrylamide or agarose gel based techniques (27). Its discriminant potential is particularly well adapted for exploring population profile changes within communities rapidly and at a very low cost, prior to amplicon sequencing $(28,29)$. However, accuracy is somehow compromised when the number of analyzed samples requires more than 1 chip, since 12 or 11 samples per chip can be analyzed for Agilent DNA 1000 and High Sensitivity chips (Agilent Technologies, Waldbronn, Germany), respectively. The discrepancies observed between electrophoregrams obtained on different chips are susceptible to bias, leading to biological misinterpretation $(16,27,30,31)$.

Several commercial software packages have been developed for fingerprint analysis, including GeneMarker (Softgenetics, State College, PA), GeneScan and GeneMapper (Applied Biosystems, Foster City, CA), and GelComparll and BioNumerics (AppliedMaths, Sint-MartensLatem, Belgium). Some free software is also available, such as GelAnalyzer (SequentiX - Digital DNA Processing, Klein Raden, Germany) or PeakScanner (Applied Biosystems). These applications all rely on peak assignment and on the presence/ absence of these peaks depending on the confidence level, which is a critical step in multiple fingerprint analysis $(16,22,28,31)$. To overcome this problem, one solution is to bin samples within windows ranging from 3 to $50 \mathrm{bp}$ (31) or to accept a $\pm 5 \%$ bp range (16).
TABLE 1. Properties and locations of the investigated soils.

\begin{tabular}{|c|c|c|c|c|}
\hline Name & Soil type & Land use & $\mathbf{p H} \mathrm{CaCl}_{2}$ & Location \\
\hline Ardallie & Peaty gley & Bog & 3.38 & $\begin{array}{l}57.4498482643 \text { latitude } \\
-2.00273413604 \text { longitude }\end{array}$ \\
\hline Rathen & Brown earth & Arable & 5.37 & $\begin{array}{l}57.6299463067 \text { latitude } \\
-2.00117490958 \text { longitude }\end{array}$ \\
\hline Drumlithie & Humus iron podzol & Grassland & 4.78 & $\begin{array}{l}56.9106598029 \text { latitude } \\
-2.32967969939 \text { longitude }\end{array}$ \\
\hline Roth & Chromic luvisol & Grassland & 4.60 & $\begin{array}{l}51.809624 \text { latitude } \\
-0.356248 \text { longitude }\end{array}$ \\
\hline Guede & Sandy soil & Arable & 7.1 & $\begin{array}{l}\text { 16.6813889 latitude } \\
-14.835 \text { longitude }\end{array}$ \\
\hline
\end{tabular}

To overcome these experimental problems and avoid missing some minor peaks, we decided to work with full fluorescence data, without DNA sizing. In this case, no software was available for correction, so we designed the RisaAligner software based on a nonlinear ladderalignment algorithm for mathematically adjusting values to a standard, enabling accurate comparison of electrophoregrams produced from different chips (with samples aligned according to upper and lower markers added to all samples on the chip, including the ladder). The mathematical correction applied to ladders to obtain a perfect match is subsequently applied to samples to allow their accurate comparison. No peak assignment of samples is required prior to profile comparison. RisaAligner also performs manual intra-chip profile re-alignment if necessary. Finally, the software includes two procedures for correcting negative fluorescent values with no biological meaning. To our knowledge, this is the first free software package that performs such a function.

\section{Reproduce Results!}

Thermal Lab Beads ${ }^{\text {TM }}$ Revolutionize Lab Baths
- Eliminate ice \& water

- Prevent inaccuracies, loss

- Keep samples organized, no floating samples

\section{Thermal Lab Beads ${ }^{\mathrm{TM}}$ diminish contamination risk}

Driven by customer needs, tecaLAB products are designed to replace traditional baths \& diminish the risk of waterborne contamination.

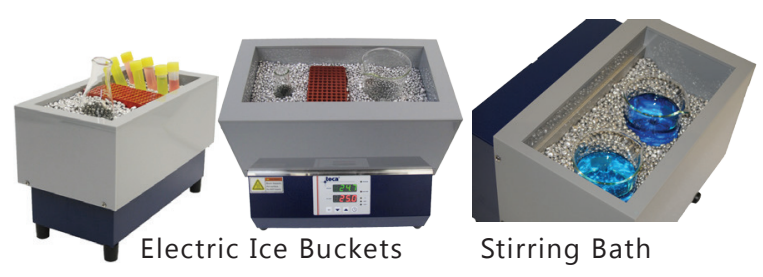

Phone 773-342-4900

Visit us www.tecalab.com 
Reference chip

\begin{tabular}{|c|c|c|c|c|c|}
\hline Data(1,k,t) & $L(1, t)$ & Data(1,k,t) & $L(1, t)$ & Data(1,k,t) & $L(1, t)$ \\
\hline $\operatorname{Data}(2, \mathrm{k}, \mathrm{t})$ & $L(2, t)$ & Data $\left(2, \mathrm{k}, \mathrm{t}^{\star}\right)$ & $\mathrm{L}\left(2, \mathrm{t}^{\star}\right)$ & Aligned Data $(2, \mathrm{k}, \mathrm{t})$ & Aligned $\mathrm{L}(2, \mathrm{t})$ \\
\hline $\operatorname{Data}(3, \mathrm{k}, \mathrm{t})$ & $L(3, t)$ & Data $\left(3, \mathrm{k}, \mathrm{t}^{\star}\right)$ & $\mathrm{L}\left(3, \mathrm{t}^{\star}\right)$ & Aligned Data $(3, \mathrm{k}, \mathrm{t})$ & Aligned $L(3, t)$ \\
\hline$\ldots$ & $\cdots$ & $\ldots$ & $\cdots$ & $\ldots$ & $\ldots$ \\
\hline Data(NL,k,t) & $L(N L, t)$ & Data(NL,k, $\left.\mathrm{t}^{\star}\right)$ & $L\left(N L, t^{\star}\right)$ & Aligned Data(NL,k,t) & Aligned $\mathrm{L}(\mathrm{NL}, \mathrm{t})$ \\
\hline
\end{tabular}

Figure 1. Illustration of the alignment algorithm. The principle of the alignment algorithm is depicted: Data( $n, k, t)=$ fluorogram for the $n$-th chips, $\mathrm{k}$-th lanes at time $t ; \mathrm{L}(n, t)=$ ladder for the $n$-th chips at time $t$. The red labels correspond to the reference chip, which is not modified during the alignment. For all of the other chips, a corrected time $t^{*}$ is first computed so that $L\left(n, t^{*}\right)$ is aligned with the reference ladder $L(1, t)$, and therefore Data $\left(n, k, t^{*}\right)$ corresponding to all lanes and chips can be compared. In a second step, an interpolation is performed in order to resample all of the data at the same time. Any further analysis will be performed on aligned data(n,k,t).

\section{Materials and methods}

Soil samples

United Kingdom (UK) and Senegal soils (Table 1) were used to demonstrate how RisaAligner software can help compare fingerprints obtained from several chips. UK soil samples were used to validate the software with DNA 1000 chips, and Senegal soil was analyzed with High Sensitivity chips due to the low content of DNA.

One of the UK soils (Rothamsted soil; collected from the Rothamsted Research Centre, Rothamsted, England, www. rothamsted.ac.uk) was used to evaluate the effect of soil storage and sampling time on microbial communities. Samplings were carried out in July 2011 (Roth_2011), July 2012 (Roth_2012), and September 2013 (Roth_ref and Roth-2013) and stored at $4{ }^{\circ} \mathrm{C}$ until extraction. The 2013 soil was extracted twice: once in September (Roth_ref) just after collection and once after 3 months of storage at $4^{\circ} \mathrm{C}$ (Roth-2013) at the same time of year as in 2011 (2.5 years of cold storage) and 2012 (1.5 years of cold storage).

Senegal bulk soil was sampled in an arid agricultural area of Guede and was incubated at $20^{\circ} \mathrm{C}$. The evolution in time of the bacterial community structure was monitored with High Sensitivity chips. Microcosms were constructed by placing $10 \mathrm{~g}$ (dry weight) of soil into $125 \mathrm{~mL}$ plasma flasks sealed with parafilm. Eight milliliters of ultra pure water was added to soil microcosms ( $90 \%$ of field capacity). Soil microcosms were kept at $20^{\circ} \mathrm{C}$ for 120 days in an incubator (Sanyo, Leicestershire, UK) under controlled conditions. The temperature was monitored with a humidity and temperature USB datalogger (IHM, Le Mans, France). Samples were taken over a period of 120 days (sampling at 0, 1, 11, 18, 32, 48, 61, 90, and 120 days). Triplicate microcosms were used for the RISA analyses.

\section{DNA extraction from soil}

DNA was extracted in triplicate from $350 \mathrm{mg}$ of the 4 UK soil samples with the NucleoSpin soil kit (Macherey-Nagel, Hoerdt, France). The first lysis buffer (SL1) was used in the presence of the enhancer (150 $\mu \mathrm{L}$ SX). The FastPrep-24 instrument (MP Biomedicals, Illkirch, France) was set to $5 \mathrm{~m} / \mathrm{s}$ for $30 \mathrm{~s}$ to lyse the samples (step 3). After step 4, the supernatant was transferred to another tube, and a second lysis was performed on soil samples with buffer SL2 without enhancer. Both supernatants for each sample were

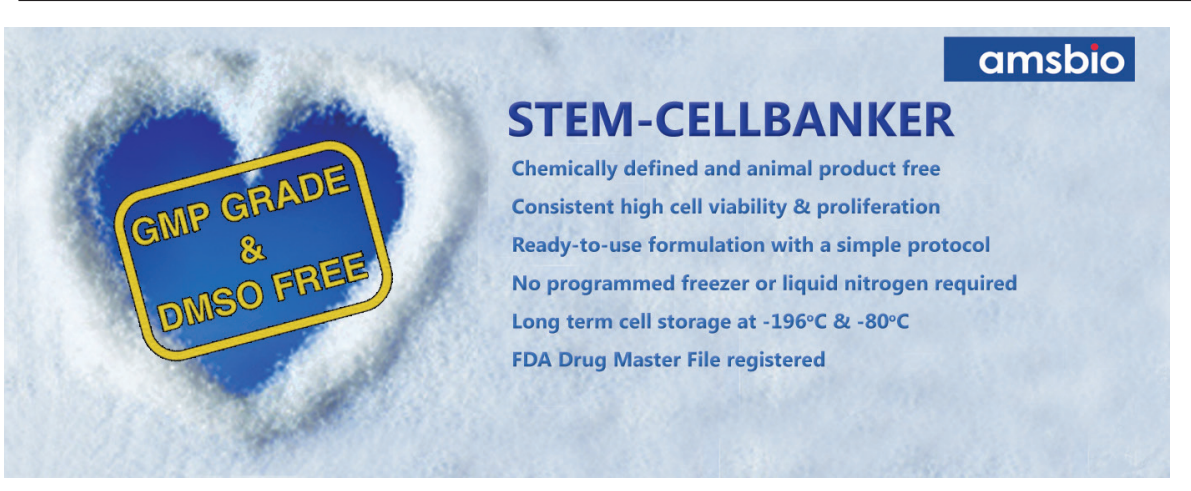

TABLE 2. Soil DNA concentrations.

\begin{tabular}{|c|c|c|}
\hline \multicolumn{2}{|c|}{ Sample name } & \multirow{2}{*}{ 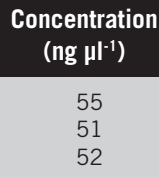 } \\
\hline Roth_ref & $\begin{array}{l}\text { R1 } \\
\text { R2 } \\
\text { R3 }\end{array}$ & \\
\hline Drumlithie & $\begin{array}{l}\text { Pz1 } \\
\text { Pz2 } \\
\text { Pz3 }\end{array}$ & $\begin{array}{l}41.8 \\
36.7 \\
41.8\end{array}$ \\
\hline Ardallie & $\begin{array}{l}\text { P1 } \\
\text { P2 } \\
\text { P3 }\end{array}$ & $\begin{array}{l}39.5 \\
43.5 \\
43.8\end{array}$ \\
\hline Rathen & $\begin{array}{l}\text { B1 } \\
\text { B2 } \\
\text { B3 }\end{array}$ & $\begin{array}{l}43.7 \\
38.5 \\
42.2\end{array}$ \\
\hline Roth_2011 & $\begin{array}{l}\text { R1 } \\
\text { R2 } \\
\text { R3 }\end{array}$ & $\begin{array}{l}60 \\
85 \\
60\end{array}$ \\
\hline Roth_2012 & $\begin{array}{l}\text { R1 } \\
\text { R2 } \\
\text { R3 }\end{array}$ & $\begin{array}{l}57 \\
59 \\
58\end{array}$ \\
\hline Roth_2013 & $\begin{array}{l}\text { R1 } \\
\text { R2 } \\
\text { R3 }\end{array}$ & $\begin{array}{l}60 \\
85 \\
60\end{array}$ \\
\hline GO (TO) & E4 & 18 \\
\hline G1 (T1) & $\begin{array}{l}\text { E16 } \\
\text { E17 } \\
\text { E18 }\end{array}$ & $\begin{array}{l}24.2 \\
22.2 \\
16.4\end{array}$ \\
\hline G2 (T11) & $\begin{array}{l}\text { E28 } \\
\text { E29 } \\
\text { E30 }\end{array}$ & $\begin{array}{c}53.6 \\
46 \\
26.2\end{array}$ \\
\hline G3 (T18) & $\begin{array}{l}\text { E40 } \\
\text { E41 } \\
\text { E42 }\end{array}$ & $\begin{array}{l}59.6 \\
39.6 \\
38.8\end{array}$ \\
\hline G4 (T32) & $\begin{array}{l}\text { E52 } \\
\text { E53 } \\
\text { E54 }\end{array}$ & $\begin{array}{c}23 \\
33.2 \\
35.8\end{array}$ \\
\hline G5 (T48) & $\begin{array}{l}\text { E64 } \\
\text { E65 } \\
\text { E66 }\end{array}$ & $\begin{array}{c}15.88 \\
31.8 \\
33.8\end{array}$ \\
\hline G6 (T61) & $\begin{array}{l}\text { E76 } \\
\text { E77 } \\
\text { E78 }\end{array}$ & $\begin{array}{c}28.8 \\
35 \\
35.6\end{array}$ \\
\hline G7 (T90) & $\begin{array}{l}\text { E88 } \\
\text { E89 } \\
\text { E90 }\end{array}$ & $\begin{array}{l}36.6 \\
16.4 \\
35.2\end{array}$ \\
\hline G8 (T120) & $\begin{array}{l}\text { E100 } \\
\text { E101 } \\
\text { E102 }\end{array}$ & $\begin{array}{l}18.3 \\
20.2 \\
21.8\end{array}$ \\
\hline
\end{tabular}


pooled and further processed according to the manufacturer's instructions. Final elution was performed with $50 \mu \mathrm{L}$ of room temperature elution buffer SE. No further purification was required.

For the soils from Senegal, DNA was extracted with the UltraClean Mega Soil DNA Isolation Kit (Mobio, Ozyme, St Quentin en Yveline, France). DNA was concentrated by ethanol precipitation and purified with the NucleoTrap Nucleic acid and protein purification Kit (Macherey-Nagel, Hoerdt, France).

DNA concentrations (Table 2) were determined using $2 \mu$ extracted DNA with the Qubit dsDNA HS Assay Kit (Invitrogen, Fisher Scientific, IIIkirch, France) on a Qubit photometer (Invitrogen).

\section{RISA}

Ribosomal intergenic sequences were amplified with primers S-D-Bact1522-b-S-20 and L-D-Bact-132-a-A-18 (22). Amplifications were obtained using the illustra Hotstart Mix RTG (GE Healthcare, Orsay, France) on $2 \mu$ of 10-fold diluted UK soil DNA or $2 \mu$ of $1 \mathrm{ng} \mathrm{\mu l}^{-1}$ diluted Senegal soil DNA (or water for the negative controls). Amplification profiles were checked on a $1 \%$ agarose gel and analyzed with the Agilent 2100 Bioanalyzer on a DNA 1000 chip (12 samples per chip) for UK soils or on a High Sensitivity chip (11 samples per chip) for Senegal soils. Before Bioanalyzer electrophoresis for Senegal samples, PCR products were purified with the NucleoSpin Gel and PCR Clean-up kit (Macherey-Nagel) according to the manufacturer's instructions.

\section{Programming}

The software, which was written in JAVA so as to ensure maximum compatibility with all types of computers, is available as a stand-alone program that can be used directly without installation. The software is protected by license (Apache 2.0: IDDN. FR.001.270024.000.R.C.2015.000.30000), and is freely available on our web site: www. ampere-lyon.fr/spip.php?article811.

Assuming that a set of chips have to be re-aligned, let $\mathrm{NL}=$ number of each chip. For each chip, the ladder $(L)$ and the sample (Data) are given, with: $\mathrm{n}$ (number of chips) $=$ 1 ... NL, $\mathrm{k}$ (number of lanes) $=1 \ldots \mathrm{NK}(\mathrm{n})$, and $\mathrm{t}(\mathrm{time})=1 \ldots \mathrm{NT}$. The alignment algorithm is composed of two steps (Figure 1). In the first step (peak matching), the algorithm compares each ladder with a reference ladder Lref(t), which can be chosen arbitrarily (by default: $\operatorname{Lref}(t)=\mathrm{L}(1, \mathrm{t})$ ). For each ladder,
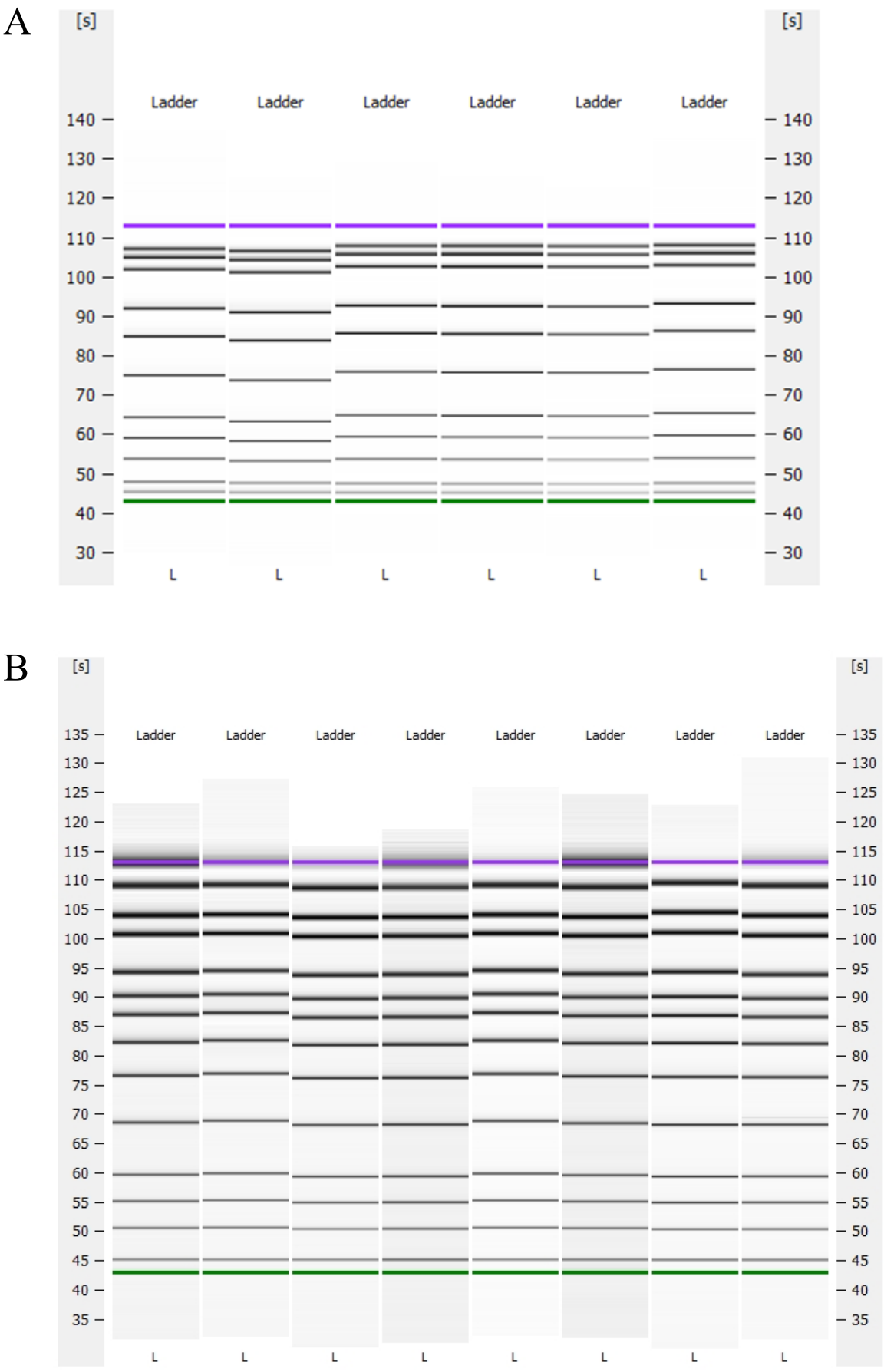

Figure 2. Comparison profiles of ladders between chips. (A) Six Agilent DNA 1000 ladders originating from six different chips were compared with the comparison option using the Agilent 2100 Expert software. (B) Eight High Sensitivity ladders originating from eight different chips were compared with the comparison option of the 2100 Expert software. The Agilent Expert software performed intra-chip alignment with upper and lower markers added to all wells but did not perform inter-chip alignment. This figure highlights migration variations among chips.

the algorithm computes a corrected time $t^{*}(n)$ so that: $L\left(n, t^{\star}(n)\right) \approx L$ ref(t). More precisely, the corrected time $\mathrm{t}^{*}(n)$ is computed by matching the times corresponding to the NP peaks of the ladders and interpolating the times between the peaks. The software can detect the ladder peaks automatically, and the user has to manually verify the correct selection of the significant peaks (Supplementary Material, "Alignment of the other ladders"). It is crucial that the number of peaks (NP) is the same for the ladders of all the chips. In the present implementation, the interpolation is performed using spine functions. (Other interpolation methods may be used as well.) For the second step (interpolation), once the 
A

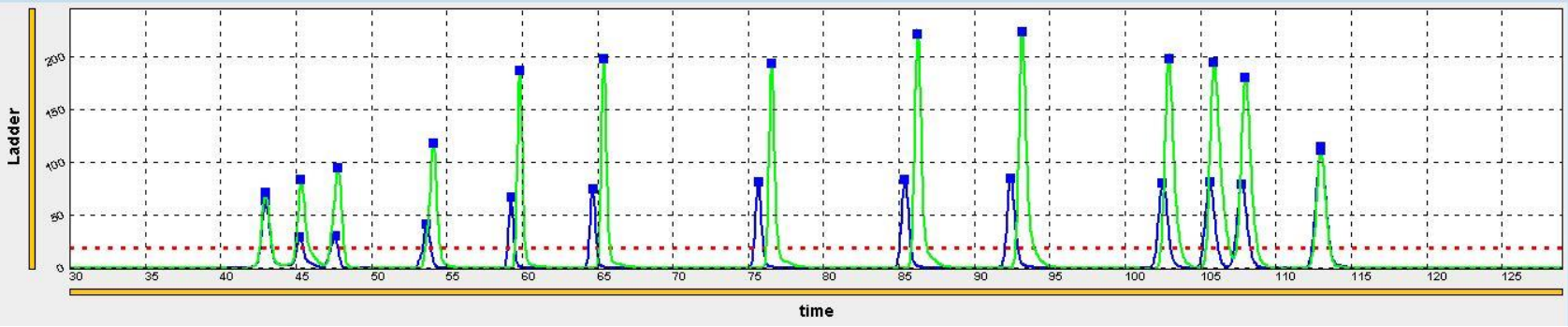

B

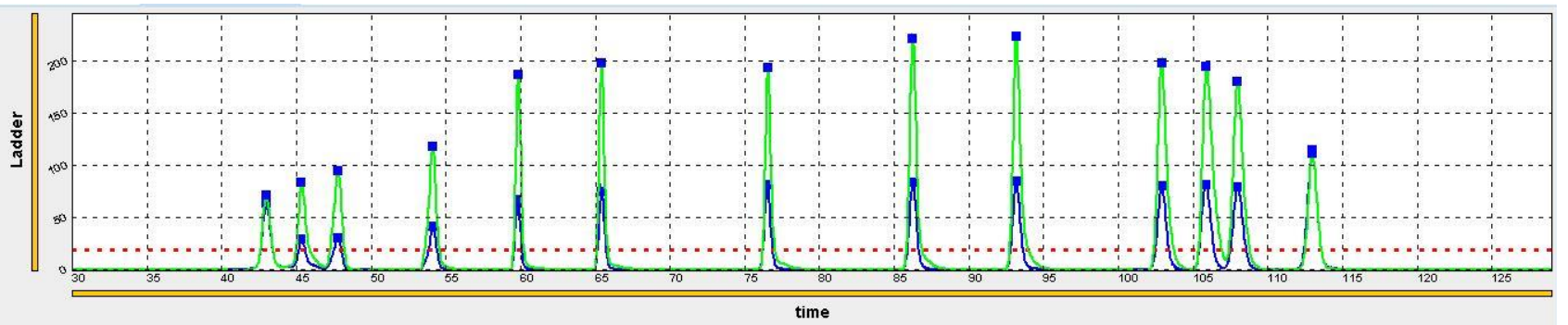

$\mathrm{C}$

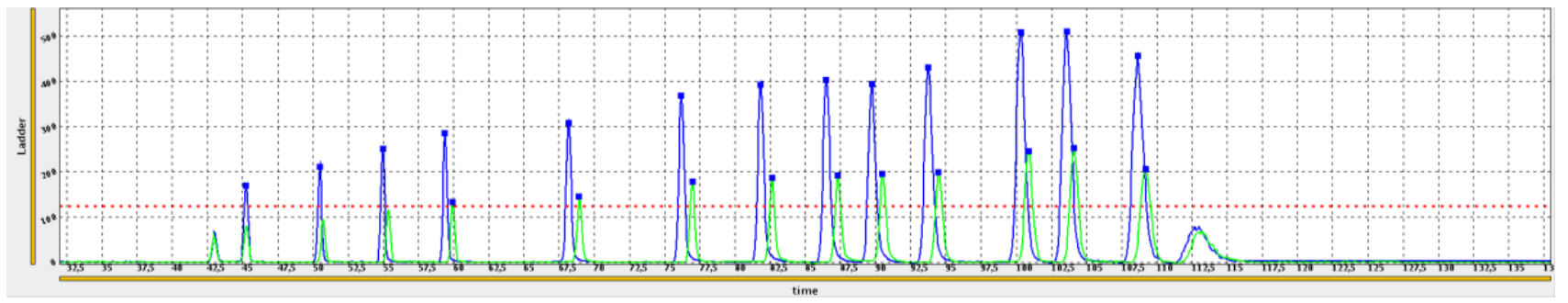

$\mathrm{D}$

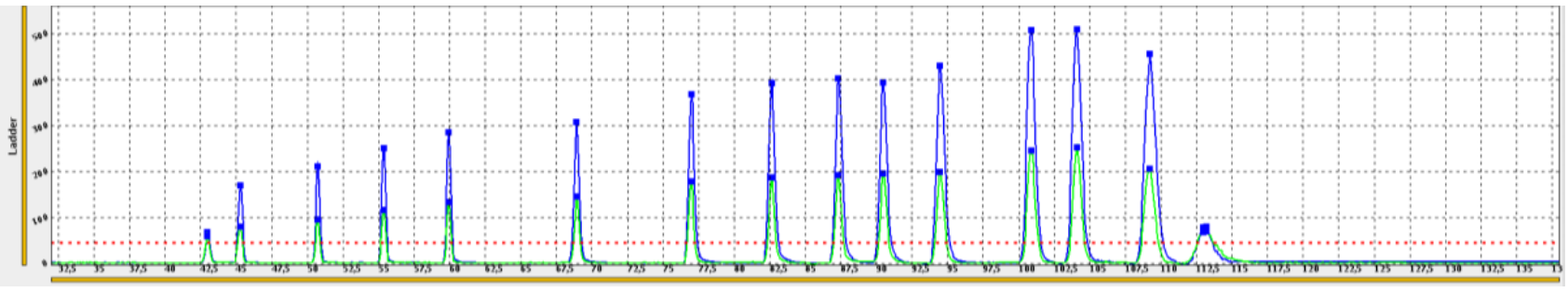

Figure 3. Alignment of ladders. (A) Unaligned ladders from a DNA 1000 chip. (B) Aligned ladders from a DNA 1000 chip. (C) Unaligned ladders from a High Sensitivity chip. (D) Aligned ladders from a High Sensitivity chip. These screen snapshots of the first step of the software procedure show the results of ladder alignment. Green: reference ladder chosen. Blue: ladder to align. Red dotted line: baseline that can be moved to detect all peaks.

corrected time $t^{*}(n)$ has been computed for the $\mathrm{n}$-th chip, the ladder of this n-th chip and of the reference chip are aligned; that is, the graphic of $L$ ref(t) is superposed with the graphic of $L\left(n, t^{*}(n)\right)$. We assume that the bias is the same for all lanes of the same chip: therefore all lanes of the chip can be aligned with respect to the reference chip by plotting Data(n,k,t*(n)) instead of Data(n,k,t). However, in order to proceed further with the analysis [for instance by principal component analysis (PCA)], all of the lanes of all of the chips must be sampled at the same time, t.
Therefore, the corresponding original lanes Data( $\left(n, k, t^{*}(n)\right)$ are interpolated to compute the corrected values at the time $t=1 \ldots$ NT. More details are available in the Supplementary Material (in the "Programming" section).

\section{Data processing}

The data processing procedure is detailed in the supporting information. In summary, folders containing .csv aligned sample data files exported from the Agilent 2100 Expert software can be directly opened by the RisaAligner software. For each data set, a tab is opened in the upper side of the main window (Supplementary Figure S1). Data can be viewed either as electrophoregrams or as gel images (Supplementary Figure S2). A reference ladder must be selected, and when all samples have the same amount of peaks detected in their respective ladders, alignments can be performed (Supplementary Figures S3S5). The aligned data can be exported as .csv files. Since original files generated by the Bioanalyzer may contain negative values 
A

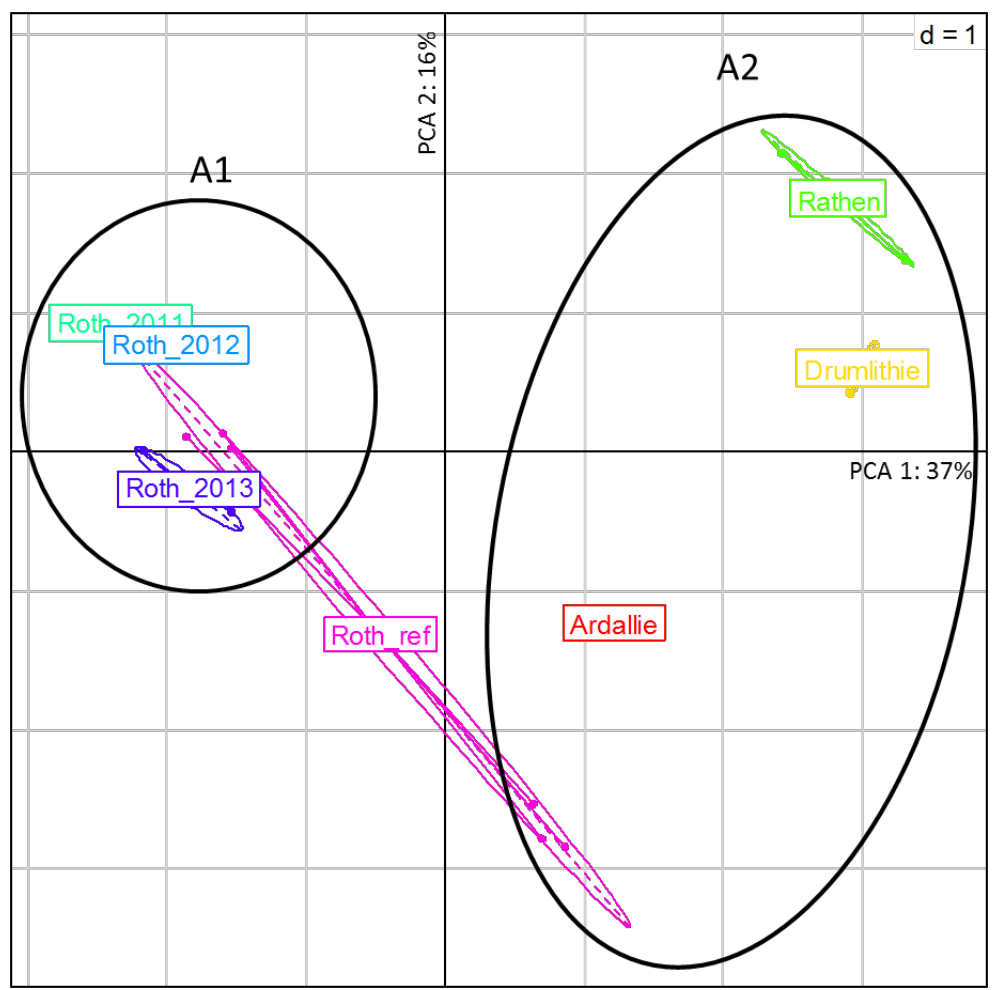

$\mathrm{B}$

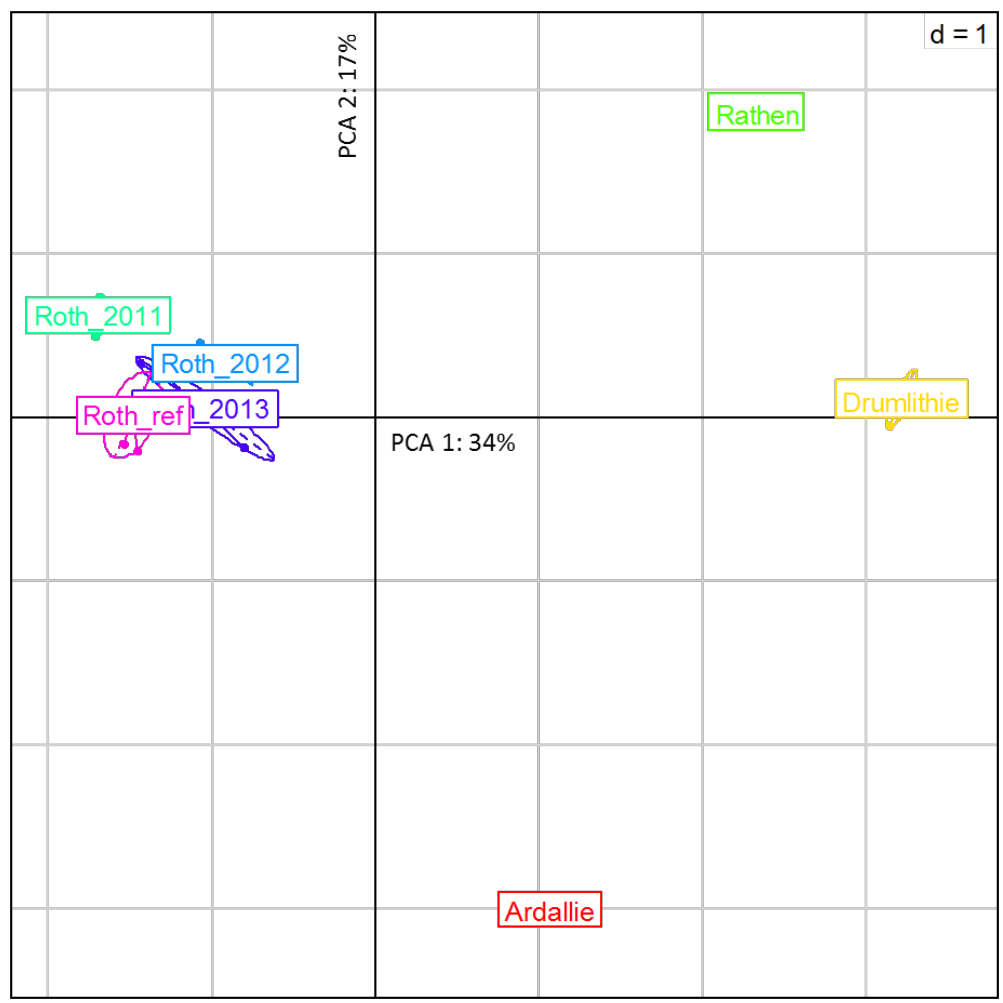

Figure 4. Principal Component Analysis (PCA) of fluorescence data from United Kingdom soils using DNA 1000 chips. (A) Raw data obtained from two chips: A1 (Roth_ref, _2011,_2012, and _2013 samples) and A2 (Roth_ref, Ardallie, Drumlithie, and Rathen samples). (B) Aligned data after software computation. Separation of Roth_ref samples by the first PCA axis according to the chip used (A1 or A2) was suppressed. Colors corresponded to the different soil samples. Pink samples (Roth_ref) were analyzed twice on two chips, A1 and A2. Three independent repetitions per soil were performed. Inertia values were obtained with the Ade4TkGUI R software package. having no biological meaning, each data set is corrected according to two procedures: (i) the extension _aligned.csv file replaces all negative values with 0 , (ii) the extension _aligned_negat.csv file shifts the horizontal axis to the lowest negative value so the minimum value becomes 0 . During the export process, the user is asked through a dialog window (Supplementary Figure S6) if the normalization must be performed on the whole data set or only on a selected range. Then, a second alignment is proposed for intra-array corrections since some samples could present identical profiles with a slight shift (Supplementary Figure S7). Similar to the primary alignment, the secondary aligned data sets can be exported as .csv files with the extensions_aligned2.csv and _aligned2_negat.csv.

\section{Statistical analyses}

Statistical analyses were performed with the Ade4TkGUI package of R (version 2.15) (32). The grouping factors were assessed with the Monte Carlo test (OrdiClust application of the ade4tkGUI package from $R$ software).

\section{Results and discussion}

The 16S-23S intergenic spacer region from the bacterial rRNA operon was amplified from the total soil microbial community DNA. RISA profiles were generated from PCR products with the Agilent 2100 Bioanalyzer using micro-fluidic technology. The Agilent 2100 Expert software plots fluorescence intensity versus size/migration time and produces an electrophoregram for each sample. Using information from a ladder with bands corresponding to precise sizes, the Agilent software generates a peak table for each sample aligned to theoretical ladder sizes. These data can be exported and further analyzed. One problem when comparing samples according to a peak table is defining which peaks are different from one another for complex profiles. The threshold varies from one study to the next $(16,22,31)$. For some applications, working with peak tables is sufficient, but for community analysis of complex samples such as soils, more information and sensitivity can be obtained with full electrophoregram data. However, reproducibility between chips cannot always be obtained due to variations in ladder migration from one chip to another. This is a major problem when several electrophoregrams have to be 
analyzed jointly without DNA sizing (Figure 2, A and B).

In order to solve this problem, we propose an alignment algorithm that corrects the data in such a way that all the ladders align, allowing meaningful comparison of the data obtained from different chips (Figure 3, A-D). More precisely, the algorithm computes a re-alignment map for each chip, which compares all of the ladders to the same single reference ladder. By applying this map to each line of the chips, the data were comparable. However the gap between the peaks of the different ladders is neither constant nor is it linear as a function of time; therefore, the re-alignment maps must be nonlinear. In our algorithm, the maps are computed using spline interpolation.

Application to a DNA 1000 chip This technique for discriminating between soil samples was evaluated using RISA on DNA 1000 chips. Four different soils from the UK were compared. For one sample, sampling was reiterated over 3 years to verify the stability of microbial communities over time, leading to the comparison of 24 profiles. One DNA 1000 chip consisted of 12 lanes, thus 2 chips were used to perform the comparison. Exported sample .csv files from the Bioanalyzer were used to perform PCA, which, as shown in Figure 4A, clearly demonstrated that the most important grouping factor was the array itself. The A1 group corresponded to the first array, and the A2 group corresponded to the second array. These separated according to the first PCA axis, which explained 37\% of the observed variability (Figure 4A). This is particularly clear in the case of the purple RISA samples (Roth_ref) that were run on both DNA 1000 chip A1 and chip A2. These separated according to the first PCA axis instead of being grouped (Figure 4A). The RisaAligner software was applied on raw data, and after re-alignment the array bias disappeared (Figure 4B) so that soil samples processed on two different chips could be directly compared. We demonstrated that soils originating from different locations (Table 1) can be statistically differentiated according to their microbial population content (Monte Carlo test, $P$-value: 0.001). This approach also demonstrated that after 4 months of cold storage (Roth_ref and Roth_2013), microbial populations were not significantly different ( $P$-value: 0.001), while sampling time $(2011,2012,2013)$ had a moderate effect on microbial community composition. (Separation according to the third axis of the
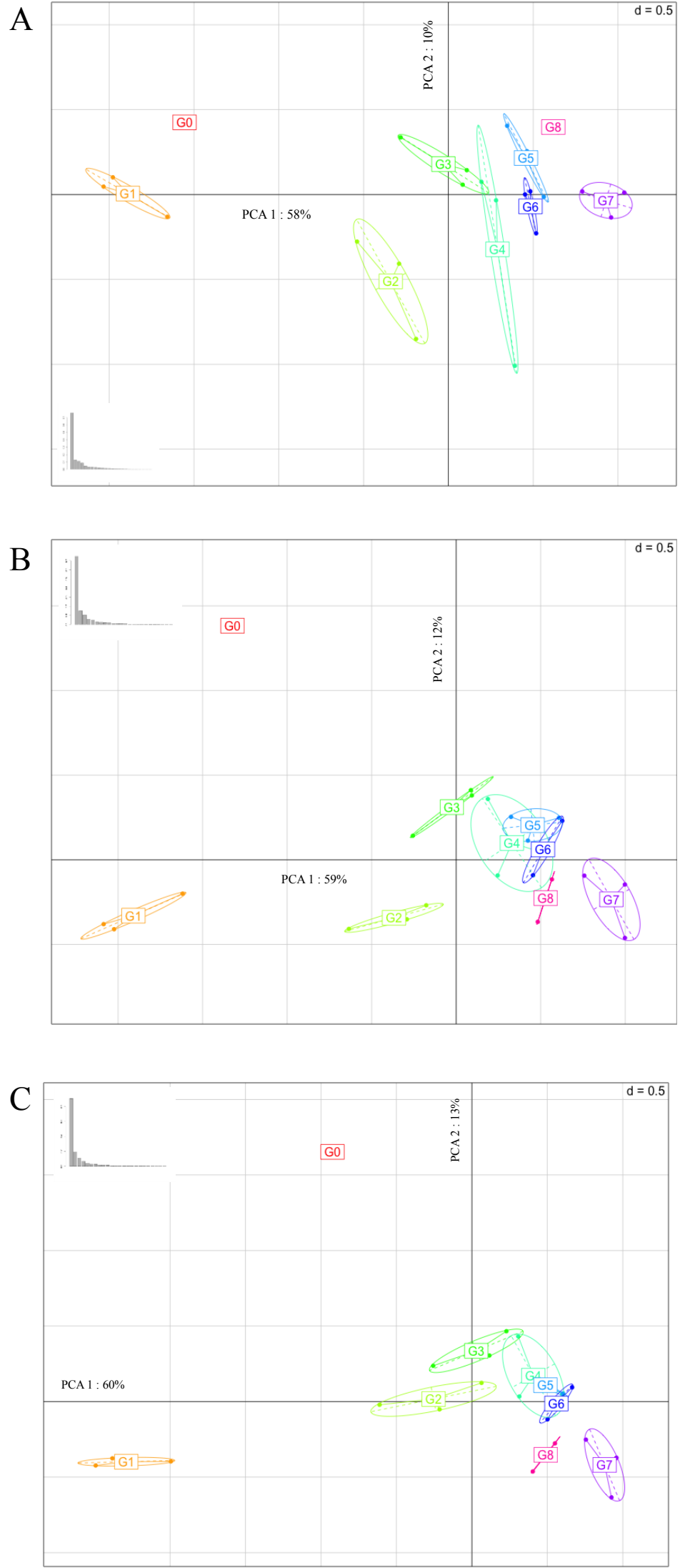

Figure 5. Principal Component Analysis (PCA) of fluorescence data of Senegal soils from High Sensitivity chips. (A) Unaligned data. (B) First step aligned data. (C) Second step aligned data. Samples are colored according to the sampling time of the soils (grouping factor). Inertia values were obtained with the Ade4TkGUI R software package. 
PCA restricted to Roth samples explained $17 \%$ of the variability; data not shown.)

Application to a High Sensitivity chip RISA on a High Sensitivity chip was used to evaluate the impact of soil incubation on bacterial community structure. Nine sampling periods (3 replicates) were performed during 120 days of incubation, leading to the comparison of 27 profiles. The samples were analyzed on 11 chips. The same artifact appeared as in the DNA 1000 chips; the G4 samples were analyzed on 2 different chips, and the most distant point showed a RISA profile performed on a different array from the 2 others (Figure 5A). This artifact was eliminated after re-alignment with the RisaAligner software (Figure 5B). A second alignment step was performed in order to improve alignment using the gel-reconstituted image. As shown in Figure 5C, this second alignment step allowed a better grouping of replicate samples similar to G5 and G6.

Application of the RisaAligner software to the soil from Guede allowed analysis of the impact of soil incubation on bacterial community structure. An important shift appeared in the profile after 1 day and a second shift at 2 days. After that, variations in bacterial community structure were smaller. The change between time 0 and 1 day could be due to temperature but also may be due to the addition of water to an arid soil, which could cause dormant bacterial populations to start growing. The same phenomena could be observed in situ at the beginning of the rainy season (data not shown).

These two examples highlight how the RisaAligner software can prevent data misinterpretation and therefore improve the determination of distances between samples.

Previously, Hewson and Fuhrman (31) developed an improved binning technique for comparisons of microbial profiles. Their technique consisted of binning all pairwise comparisons in multiple bin window frames, with the starting size of the window (i.e., frame) shifted by +1 bp for a total of $x$ frames, where $x$ bp is the width of the maximum bin window size in any binning scheme (31). This technique was further refined by Wilkinson (27) to address the risk of artifact introduction by splitting one unit between two bins, resulting in an underestimation of similarity. However, these approaches were developed to reduce size calling imprecision and did not allow alignment of full electro- phoregrams by considering all fluorescent values from 30 to $110 \mathrm{~s}$.

The RisaAligner software was not developed to increase size assignment accuracy of each band, but instead was created for comparison of full fluorescence electrophoregrams (aligned sample data) exported from the Agilent 2100 Expert software. It is not limited by the number of chip comparisons and allows the analysis of a large number of samples on different chips. This program can be applied to other fingerprinting methods performed on automatic apparatuses providing numerical data, such as randomly amplified polymorphic DNA (RAPD) analysis, BOX-PCR, or restriction fragment length polymorphism (RFLP) analysis with DNA 7500 chips on the Agilent 2100 Bioanalyzer $(30,33)$. RisaAligner was programmed in JAVA to ensure maximum compatibility with all kind of computers. This tool can be freely downloaded at www. ampere-lyon.fr/spip.php?article811.

\section{Author contributions}

O. F., R.S., E.N., and S.D. developed the program. E.N., J.R., and S.D. performed the experiments. O.F., R.S., E.N., S.D.,

High speed modulation

The new 56-CRN, 57-CFS, and 57-CFP series diode laser systems from Melles Griot provide excellent beam performance and pointing stability from a compact, self-contained package. This industry standard $70 \mathrm{~mm} \times 40 \mathrm{~mm} \times 40 \mathrm{~mm}$ package is a must for your OEM application by incorporating analog and digital drive electronics to provide a smaller footprint at a reduced cost.

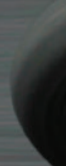
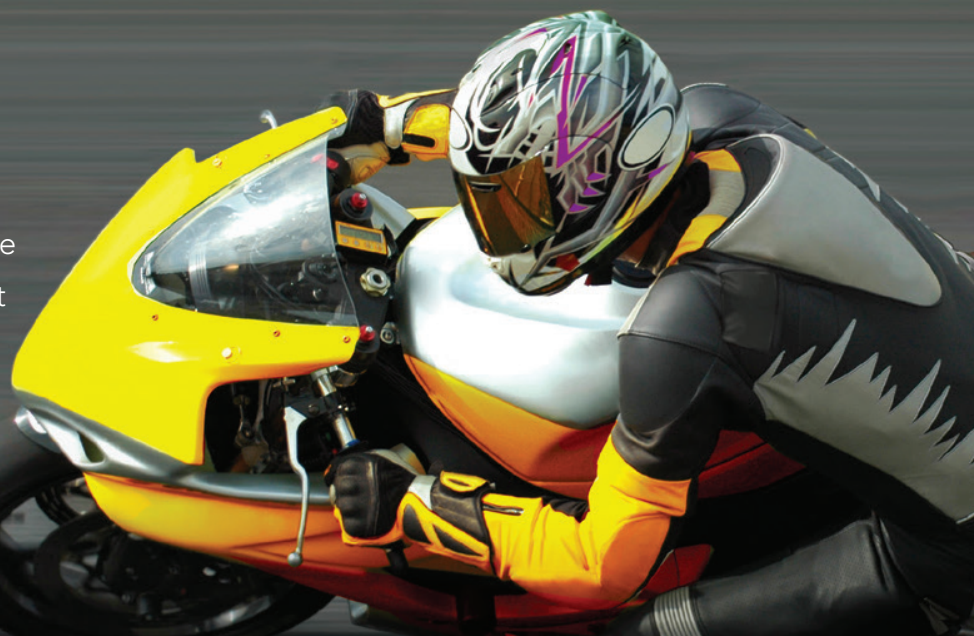
s. 
P.S., and L.D. wrote the manuscript. S.D. conceptualized the study.

\section{Acknowledgments}

Research reported in this work was supported by the European Union under grant agreement number 313149 (MiSAFE project, European FP7 Theme 10: Security Call: FP7-SEC-2012-1; http:// forensicmisafe.wix.com/misafe) and by the French government "Agence Nationale de la Recherche" under the reference ANR-08-VULN-011 (SOLAO project, ANR VMCS). Many thanks to the Rothamsted research center for permission to sample the grassland soil.

The authors declare no competing interests.

\section{References}

1. Delmont, T.O., P. Simonet, and T.M. Vogel. 2012. Describing microbial communities and performing global comparisons in the 'omic era. ISME J. 6:1625-1628.

2. Daniel, R. 2005. The metagenomics of soil. Nat. Rev. Microbiol. 3:470-478.

3. Handelsman, J. 2004. Metagenomics: Application of Genomics to Uncultured Microorganisms. Microbiol. Mol. Biol. Rev. 68:669-685.

4. Courtois, S., C.M. Cappellano, M. Ball, F.X. Francou, P. Normand, G. Helynck, A. Martinez, S.J. Kolvek, et al. 2003. Recombinant environmental libraries provide access to microbial diversity for drug discovery from natural products. Appl. Environ. Microbiol. 69:49-55.

5. Demanèche, S., M.M. David, E. Navarro, P. Simonet, and T.M. Vogel. 2009. Evaluation of functional gene enrichment in a soil metagenomic clone library. J. Microbiol. Methods 76:105-107.

6. Jacquiod, S., S. Demanèche, L. Franqueville, L. Ausec, Z. Xu, T.O. Delmont, V. Dunon, C. Cagnon, et al. 2014. Characterization of new bacterial catabolic genes and mobile genetic elements by high throughput genetic screening of a soil metagenomic library. J. Biotechnol. 190:18-29.

7. Bauer, J.D., R.W. King, and S.F. Brady. 2010. Utahmycins a and B, azaquinones produced by an environmental DNA clone. J. Nat. Prod. 73:976-979.

\section{Competing interests}

8. Meyer, F., D. Paarmann, M. D'Souza, R. Olson, E.M. Glass, M. Kubal, T. Paczian, A. Rodriguez, et al. 2008. The metagenomics RAST server - a public resource for the automatic phylogenetic and functional analysis of metagenomes. BMC Bioinformatics 9:386.

9. Prakash, T. and T.D. Taylor. 2012. Functional assignment of metagenomic data: challenges and applications. Brief. Bioinform. 13:711-727.

10. Delmont, T.O., C. Malandain, E. Prestat, C. Larose, J.-M. Monier, P. Simonet, and T.M. Vogel. 2011. Metagenomic mining for microbiologists. ISME J. 5:1837-1843.

11. Dröge, J. and A.C. McHardy. 2012. Taxonomic binning of metagenome samples generated by next-generation sequencing technologies. Brief. Bioinform. 13:646-655.

12. Shokralla, S., J.L. Spall, J.F. Gibson, and M. Hajibabaei. 2012. Next-generation sequencing technologies for environmental DNA research. Mol. Ecol. 21:1794-1805.

13. Gilbert, J.A., J.K. Jansson, and R. Knight. 2014. The Earth Microbiome project: successes and aspirations. BMC Biol. 12:69.

14. Campisano, A., L. Antonielli, M. Pancher, S. Yousaf, M. Pindo, and I. Pertot. 2014. Bacterial endophytic communities in the grapevine depend on pest management. PLoS ONE 9:e112763.

15. Cardinale, M., L. Brusetti, P. Quatrini, S. Borin, A.M. Puglia, A. Rizzi, E. Zanardini, C. Sorlini, et al. 2004. Comparison of different primer sets for use in automated ribosomal intergenic spacer analysis of complex bacterial communities. Appl. Environ. Microbiol. 70:6147-6156.

16. Ciesielski, S., K. Bułkowska, D. Dabrowska, D. Kaczmarczyk, P. Kowal, and J. Mozejko. 2013. Ribosomal intergenic spacer analysis as a tool for monitoring methanogenic Archaea changes in an anaerobic digester. Curr. Microbiol. 67:240-248.

17. Corneo, P.E., A. Pellegrini, L. Cappellin, C. Gessler, and I. Pertot. 2013. Weeds influence soil bacterial and fungal communities. Plant Soil 373:107-123.

18. Fechner, L.C., F. Vincent-Hubert, P. Gaubert, T. Bouchez, C. Gourlay-Francé, and M.-H. Tusseau-Vuillemin. 2010. Combined eukaryotic and bacterial community fingerprinting of natural freshwater biofilms using automated ribosomal intergenic spacer analysis. FEMS Microbiol. Ecol. 74:542-553.

19. Hartmann, M., B. Frey, R. Kölliker, and F. Widmer. 2005. Semi-automated genetic analyses of soil microbial communities: comparison of T-RFLP and RISA based on descriptive and discriminative statistical approaches. J. Microbiol. Methods 61:349-360.

20. Jones, S.E., A.L. Shade, K.D. McMahon, and A.D. Kent. 2007. Comparison of primer sets for use in automated ribosomal intergenic spacer analysis

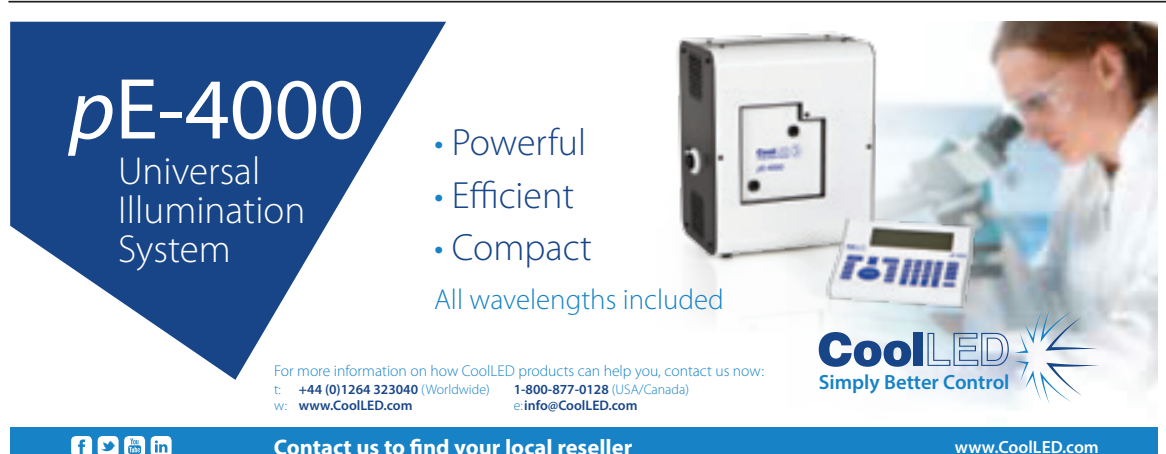

of aquatic bacterial communities: an ecological perspective. Appl. Environ. Microbiol. 73:659-662.

21. Pancher, M., M. Ceol, P.E. Corneo, C.M.O. Longa, S. Yousaf, I. Pertot, and A. Campisano. 2012. Fungal endophytic communities in grapevines (Vitis vinifera L.) respond to crop management. Appl. Environ. Microbiol. 78:4308-4317.

22. Ranjard, L., F. Poly, J.C. Lata, C. Mougel, J. Thioulouse, and S. Nazaret. 2001. Characterization of bacterial and fungal soil communities by automated ribosomal intergenic spacer analysis fingerprints: biological and methodological variability. Appl. Environ. Microbiol. 67:4479-4487.

23. Borneman, J. and E.W. Triplett. 1997. Molecular microbial diversity in soils from eastern Amazonia: evidence for unusual microorganisms and microbial population shifts associated with deforestation. Appl. Environ. Microbiol. 63:2647-2653.

24. Héry, M., S. Nazaret, T. Jaffré, P. Normand, and E. Navarro. 2003. Adaptation to nickel spiking of bacterial communities in neocaledonian soils. Environ. Microbiol. 5:3-12.

25. Ranjard, L., S. Nazaret, F. Gourbière, J. Thioulouse, P. Linet, and A. Richaume. 2000. A soil microscale study to reveal the heterogeneity of $\mathrm{Hg}(\mathrm{II})$ impact on indigenous bacteria by quantification of adapted phenotypes and analysis of community DNA fingerprints. FEMS Microbiol Ecol. 31:107-115.

26. Corneo, P.E., A. Pellegrini, L. Cappellin, M. Roncador, M. Chierici, C. Gessler, and I. Pertot. 2013. Microbial community structure in vineyard soils across altitudinal gradients and in different seasons. FEMS Microbiol. Ecol. 84:588-602.

27. Wilkinson, P.G. 2011. Characterisation of the bacterial flora associated with the grey field slug Deroceras reticulatum and assessment of its suitability as a target for biological control. Thesis. The University of Edingurgh.

28. Gobet, A., A. Boetius, and A. Ramette. 2014 Ecological coherence of diversity patterns derived from classical fingerprinting and Next Generation Sequencing techniques. Environ. Microbiol. 16:2672-2681.

29. Newsome, L., K. Morris, D. Trivedi, N. Atherton, and J.R. Lloyd. 2014. Microbial reduction of uranium(VI) in sediments of different lithologies collected from Sellafield. Appl. Geochem. 51:55-64.

30. Nachamkin, I., N.J. Panaro, M. Li, H. Ung, P.K. Yuen, L.J. Kricka, and P. Wilding. 2001. Agilent 2100 Bioanalyzer for Restriction Fragment Length Polymorphism Analysis of the Campylobacter jejuni Flagellin Gene. J. Clin. Microbiol. 39:754-757.

31. Hewson, I. and J.A. Fuhrman. 2006. Improved strategy for comparing microbial assemblage fingerprints. Microb. Ecol. 51:147-153.

32. Thioulouse, J., S. Dray, et al. 2007. Interactive Multivariate Data Analysis in $\mathrm{R}$ with the ade4 and ade4TkGUI Packages. J. Stat. Softw. 22:1-14.

33. Fothergill, J.L., J. White, J.E. Foweraker, M.J. Walshaw, M.J. Ledson, E. Mahenthiralingam, and C. Winstanley. 2010. Impact of Pseudomonas aeruginosa Genomic Instability on the Application of Typing Methods for Chronic Cystic Fibrosis Infections. J. Clin. Microbiol. 48:2053-2059.

Received 07 May 2015; accepted 28 August 2015.

Address correspondence to Sandrine Demaneche, Laboratoire Ampère, Ecole Centrale de Lyon, Ecully, France. E-mail: sandrine.demaneche@ec-lyon.fr 